\title{
31 gene expression-based signatures serve as indicators of prognosis for patients with glioma
}

\author{
ZHONGJUN YAN $^{1 *}$, JIANLONG YANG $^{2 *}$, LINGLING FAN $^{3 *}$, DONGWEI XU ${ }^{1}$ and YAN HU ${ }^{1}$ \\ ${ }^{1}$ Neurosurgery Department, The Second Affiliated Hospital of The Fourth Military Medical University, \\ Xi'an, Shanxi 710038; ${ }^{2}$ Neurosurgery Department, The First Hospital of Yulin, Yulin, Shanxi 719000; \\ ${ }^{3}$ Neurology Department, The First Affiliated Hospital of Xi'an Medical University, Xi'an, Shanxi 710077, P.R. China
}

Received December 24, 2017; Accepted July 17, 2018

DOI: $10.3892 / 01.2019 .10327$

\begin{abstract}
Glioma has one of the highest mortality rates of all cancer types; however, the prognosis cannot be predicted effectively using clinical indicators, due to the biological heterogeneity of the disease. A total of 31 gene expression-based signatures were identified using selected features in The Cancer Genome Atlas cohorts and machine learning methods. The signatures were assayed in the training dataset and were further validated in four completely independent datasets. Association analyses were implemented, and the results indicated that the signature was not significantly associated with age, radiation therapy or primary tumor size. A nomogram for the 1-year overall survival rate of patients with glioma following initial diagnosis was plotted to facilitate the clinical utilization of the signature. Gene Set Enrichment Analysis was performed based on the signature, in order to determine the potential altered pathways. Metabolic pathways were determined to be significantly enriched. In summary, the 31 gene expression-based signatures were effective and robust in predicting the clinical outcome of glioma in 1,016 glioma samples in five independent international cohorts.
\end{abstract}

\section{Introduction}

It has been reported that there are $\sim 3.19$ glioma cases in every 100,000 people in USA (1). Similar trends were reported in China (2), with $>101,600$ new glioma cases and $>61,100$ glioma-associated mortalities were estimated in 2015; however, the clinical outcome of glioma is unfavorable, with a 5 -year overall survival rate of $<5 \%$. Although clinical

Correspondence to: Dr Yan Hu, Neurosurgery Department, The Second Affiliated Hospital of The Fourth Military Medical University, 1 Xinsi Road, Xi'an, Shaanxi 710038, P.R. China

E-mail: huayan_xian@163.com

${ }^{*}$ Contributed equally

Key words: prognosis, glioma, expression indicators have provided beneficial information $(3,4)$, determining the prognosis of patients with glioma remains notably difficult, due to genetic differences between individuals $(5,6)$. Isocitrate dehydrogenase $[\mathrm{NADP}(+)] 1 / 2$ mutations have been emphasized as prognostic biomarkers over the past decade and have been used clinically (7-9). Nevertheless, single mutational biomarkers remain inadequate for prognosis prediction.

Previous studies have screened single biomarkers for the prognosis of glioma $(10,11)$. It has been reported that the mRNA and protein expression of RAB34 are notably associated with poor survival rates in patients with glioma (12). Similarly, overexpression of Roundabout4 has been demonstrated to predict a poor clinical outcome of glioma by affecting micro-vessel density (13). microRNAs (miRs), including miR-34a, have been reported to be correlated with the survival of patients with glioma (14). Nevertheless, none of the aforementioned biomarkers, except for IDH1/2 mutation, have been used clinically, and the biomarkers currently utilized in the clinic remain limited. Multiple gene-based prognostic models, which combine information from single biomarkers and effectively remove the redundant information of genomes/transcriptomes, have been reported (15-17). Clinically used models, including Mammaprint and OncotypeDX, have been beneficial tools for prognosis and therapy guidance (18-20); however, to the best of our knowledge, these models have not been employed to determine the prognosis of glioma to date.

In the present study, the prognostic genes in glioma were selected by associating gene expression and overall survival in The Cancer Genome Atlas (TCGA) cohort, and the prognostic effect was further validated in 1,016 samples across five independent datasets. The signatures were not significantly associated with clinical indicators, and associated biological pathways were therefore identified.

\section{Materials and methods}

Data pre-processing. The primary glioma samples from TCGA, GSE4412 (21), GSE16011 (22), GSE16581 (23) and GSE42669 (24) were used in the present study, whilst the other sample types, with the exception of primary glioma, were excluded. The gene expression data from the TCGA cohort (evaluated using RNA-seq technique) was downloaded from the official website of TCGA (https://gdc-docs.nci.nih.gov/). 
Following upper-quantile normalization, the FPKM values of each gene was $\log 2$ transformed. The cohorts generated from microarray (GSE4412, GSE16011, GSE16581 and GSE42669) were downloaded from the Gene Expression Omnibus (https://www.ncbi.nlm.nih.gov/geo) website using the corresponding accession number. The raw data were normalized using limma package of R software in a single cohort, and a gene expression matrix was generated, according to the raw data, where rows represented the genes and columns represented the samples. To reduce the complexity, probes representing the same gene were calculated using the mean average value. To eliminate the bias brought by the platforms, z-scores were calculated in each cohort for subsequent analyses.

Optimizing panel and model development. Cox univariate regression was implemented by associating the overall survival and gene expression information of the TCGA $(n=529)$ dataset. Genes significantly associated with overall survival $(\mathrm{P}<0.01)$ were selected as candidate genes for model development. Redundant information existed among the candidate genes, due to complex regulations. To narrow the panel, the redundant information was removed, utilization was facilitated, and a machine learning algorithm called random forest variable selection method was used in this step. Using the selected 31 genes generated (Table I), Cox multivariate regression was performed by associating the overall survival and expression of these 31 genes. The coefficient of each gene is listed in Table I.

Gene panel optimization and model development. Expression of genes significantly associated with overall survival, as determined by Cox univariate regression $(\mathrm{P}<0.01)$, were identified for further analysis in the TCGA cohort. The random forest variable selection was conducted using the following parameters: 100 iterations and 100 repeats. Subsequently, the top selected gene list (panel) using this random forest survival algorithm was optimized for model development. The model was developed using Cox multivariate regression by associating the optimized panel and overall survival information in the TCGA cohort. The glioma risk score was calculated using the following formula:

$$
\text { Risk score }=\sum_{i}^{n} b_{i} * e_{i}
$$

Where $b_{i}$ is the coefficient, and $e_{i}$ is the relative expression of corresponding gene.

Statistical analysis. All analyses listed in the present study were implemented on an $\mathrm{R}$ programming platform (www.r-project.org; v3.2.0). The clinical information of samples in TCGA cohort was listed as following: 439 received radiation and 98 did not. The median age was 59 years old (1st-3rd quartile, 50-68 years, and 60 was used as a cut-off value); 235 females and 375 males. Genes significantly associated with survival were identified using Cox univariate regression $(\mathrm{P}<0.01)$, and the risk score was calculated using Cox multivariate regression in TCGA cohort. The Gene Set Enrichment Analysis (GSEA) was implemented on the publicly released GSEA 3.0 software (25). The nomogram was plotted with the R package 'rms', and random forest variable selection was implemented with the package 'randomforestSRC' $(26,27)$. The receiving operating characteristic (ROC) curve was calculated using the package 'pROC' (28). Kaplan-Meier method and log-rank test was used to determine the survival difference between high-risk and low-risk groups. Association between risk score and clinical indicators were assayed using unpaired Student's t-test. $\mathrm{P}<0.05$ was considered to indicate a statistically significant difference.

\section{Results}

Prognostic value of SACH-CASS in the TCGA cohort. The prognostic performance of the signatures was assessed in the TCGA cohort. The samples of the TCGA cohort were divided into high-score and low-score groups, according to the median risk score value, and the survival difference between the high- and low-risk groups was later compared. The patients in the low-score group had a significantly (Kaplan-Meier test, $\mathrm{P}=0.00048$ ] reduced overall survival time [median survival time, 12.7 months; $95 \%$ confidence interval (CI), 11.7-15.1 months), compared with the patients in the high-score group (median survival time, 14.9 months, 95\% CI, 14.0-16.9 months; Fig. 1A). The disease-free survival difference between the high and low-risk groups were also compared. The low-score group had significantly increased disease-free survival time, compared with the high-score group (Fig. 1B; $\mathrm{P}<0.05$ ). As depicted in Fig. 1C, low-score samples were characterized by early incidence, high expression of glioma-promoting genes and low expression of glioma-suppressing genes (middle panel, red dots). Taken together, these results indicated that the glioma scores are important and beneficial clinical indicators for glioma in the TCGA cohort.

Robustness of risk score. Due to the model being developed based on expression in the TCGA cohort, the validity of the model may result from over-fitness, as the model may only be effective in the TCGA cohort. Therefore, in order to evaluate the robustness of the risk score in an unbiased manner, The coefficients of these 31 genes were locked, therefore, the coefficients of these genes were identical to the TCGA cohort. the risk score of each sample in the validation cohorts (expression data were generated using microarray) was calculated, including the samples from the following: GSE4412, GSE16011, GSE16581 and GSE42669 cohorts. Similarly, to the training cohort, the samples in each cohort were subsequently divided into high-score and low-score groups according to the median glioma risk score, and the survival difference between high-score and low-score groups was compared (Fig. 2A-D). As expected, the gene expression pattern was similar to that of the TCGA cohort (Fig. 2E-H). Taken together, the aforementioned results indicated that the glioma risk scores are robust in predicting glioma survival across cohorts and platforms.

Existing clinicopathological indicators and glioma risk score. Associations between glioma risk scores and clinicopathological indicators, including age, tumor size, radiation were analyzed by comparing the glioma risk scores 
Table I. Parameters of candidate genes.

\begin{tabular}{|c|c|c|c|c|c|c|}
\hline \multirow[b]{2}{*}{ Entrez_ID } & \multicolumn{3}{|c|}{ Cox univariate } & \multicolumn{3}{|c|}{ Cox multivariate } \\
\hline & HR & $95 \% \mathrm{CI}$ & P-value & HR & $95 \% \mathrm{CI}$ & P-value \\
\hline 6383 & 1.2 & $1.1-1.3$ & $0.00032^{\mathrm{a}}$ & 0.96 & 0.84-1.1 & 0.547 \\
\hline 4493 & 1.2 & $1.1-1.3$ & $8 \times 10^{5 a}$ & 1.05 & $0.93-1.18$ & 0.433 \\
\hline 4223 & 1.1 & 1-1.1 & $0.00036^{\mathrm{a}}$ & 1.00 & $0.93-1.07$ & 0.948 \\
\hline 4016 & 1.1 & $1.1-1.2$ & $0.00027^{\mathrm{a}}$ & 1.05 & $0.94-1.17$ & 0.411 \\
\hline 9922 & 1.4 & $1.1-1.6$ & $0.00031^{\mathrm{a}}$ & 0.97 & $0.76-1.23$ & 0.777 \\
\hline 3383 & 1.2 & $1.1-1.3$ & $0.00085^{\mathrm{a}}$ & 0.88 & $0.72-1.08$ & 0.217 \\
\hline 1116 & 1.1 & $1.1-1.1$ & $1 \times 10^{5 \mathrm{a}}$ & 0.98 & $0.9-1.07$ & 0.725 \\
\hline 9332 & 1.1 & 1-1.2 & $4 \times 10^{4 a}$ & 1.03 & $0.94-1.13$ & 0.492 \\
\hline 716 & 1.1 & $1.1-1.2$ & $3 \times 10^{5 a}$ & 1.06 & $0.94-1.2$ & 0.311 \\
\hline 622 & 1.3 & $1.1-1.5$ & $3 \times 10^{5 a}$ & 1.14 & $0.95-1.37$ & 0.153 \\
\hline 597 & 1.1 & $1.1-1.2$ & $0.00076^{\mathrm{a}}$ & 1.06 & $0.92-1.23$ & 0.385 \\
\hline 10630 & 1.1 & $1.1-1.2$ & $<0.001^{\mathrm{a}}$ & 1.02 & $0.91-1.14$ & 0.727 \\
\hline 4615 & 1.3 & $1.1-1.5$ & $0.00015^{\mathrm{a}}$ & 0.86 & $0.68-1.1$ & 0.236 \\
\hline 3964 & 1.3 & $1.1-1.4$ & $1 \times 104^{a}$ & 1.07 & $0.84-1.37$ & 0.574 \\
\hline 3669 & 1.2 & $1.1-1.3$ & $0.00045^{\mathrm{a}}$ & 1.01 & $0.87-1.18$ & 0.855 \\
\hline 487 & 0.32 & $0.18-0.56$ & $7 \times 10^{5 a}$ & 0.44 & $0.22-0.89$ & $0.022^{\mathrm{a}}$ \\
\hline 7253 & 0.67 & $0.53-0.84$ & $0.00072^{\mathrm{a}}$ & 0.8 & $0.62-1.03$ & 0.080 \\
\hline 7132 & 1.2 & 1.1-1.4 & $0.00046^{\mathrm{a}}$ & 0.8 & $0.63-1$ & $0.049^{\mathrm{a}}$ \\
\hline 6988 & 1.3 & $1.1-1.5$ & $8 \times 10^{5 a}$ & 1.2 & $0.97-1.48$ & 0.085 \\
\hline 11000 & 1.2 & $1.1-1.3$ & $4 \times 10^{5 a}$ & 0.99 & $0.86-1.15$ & 0.911 \\
\hline 6279 & 1.1 & 1-1.2 & $0.00042^{\mathrm{a}}$ & 1.04 & $0.93-1.17$ & 0.499 \\
\hline 5154 & 1.2 & $1.1-1.2$ & $0.00017^{\mathrm{a}}$ & 1.06 & 0.94-1.19 & 0.367 \\
\hline 4599 & 1.1 & 1-1.2 & $0.00091^{\mathrm{a}}$ & 1.05 & $0.96-1.15$ & 0.264 \\
\hline 4478 & 1.3 & 1.1-1.4 & $1 \times 10^{5 \mathrm{a}}$ & 0.92 & $0.75-1.11$ & 0.378 \\
\hline 3281 & 0.73 & $0.61-0.87$ & $0.00049^{\mathrm{a}}$ & 0.62 & $0.49-0.77$ & $3.00 \mathrm{E}-05^{\mathrm{a}}$ \\
\hline 8324 & 1.2 & 1.1-1.3 & $1 \times 10^{5 a}$ & 1.05 & $0.93-1.19$ & 0.433 \\
\hline 6990 & 1.3 & $1.1-1.4$ & $<0.001^{\mathrm{a}}$ & 1.06 & $0.88-1.28$ & 0.556 \\
\hline 4192 & 1.2 & $1.1-1.3$ & $3 \times 10^{5 a}$ & 1.06 & $0.94-1.21$ & 0.343 \\
\hline 2887 & 1.2 & $1.1-1.3$ & $0.001^{\mathrm{a}}$ & 1 & $0.87-1.13$ & 0.953 \\
\hline 11068 & 1.4 & $1.2-1.7$ & $0.00052^{\mathrm{a}}$ & 0.94 & $0.68-1.31$ & 0.729 \\
\hline 1819 & 1.5 & $1.3-1.8$ & $<0.001^{\mathrm{a}}$ & 1.28 & $0.97-1.7$ & 0.086 \\
\hline
\end{tabular}

${ }^{\mathrm{a}}<0.05$. HR, hazard ratio; $95 \% \mathrm{CI}, 95 \%$ confidence interval.

in different sub-categories. The results indicated that the glioma risk scores were not clinicopathological indicators for age ( $\mathrm{P}>0.05$; cut-off, 60 years), radiation therapy or primary tumor size (cut-off:10 mm; Fig. 3A; Student's t-test). To evaluate the clinical significance of clinical indicators and glioma risk scores, Cox multivariate regression was implemented, and the results indicated that the glioma scores are an important indicator for overall survival (Fig. 3B). To facilitate the utilization of other clinical indicators and glioma risk scores, a 1-year overall survival nomogram was calculated (Fig. 3C). In summary, these results indicated that the glioma scores are an important indicator for the prognosis of patients with glioma.

Biological relevance of risk score. Due to the signatures being developed based on the expression level of 31 genes and the glioma scores being independent of the majority of clinical indicators, it was suspected that the glioma scores predicted the survival of glioma via reflecting the biological differences among individuals; therefore, GSEA was performed by comparing the difference between the high- and low-score groups. As expected, pathways, including terpenoid backbone biosynthesis, glycosphingolipid biosynthesis lacto and neolacto series, were identified (Fig. 4). In summary, the results indicated that the glioma risk score may be associated with metabolic pathways in glioma and therefore predict the survival of patients with glioma.

\section{Discussion}

Genetic alterations of glioma have been frequently reported previously $(8,9,29,30)$. Genetic heterogeneity across 

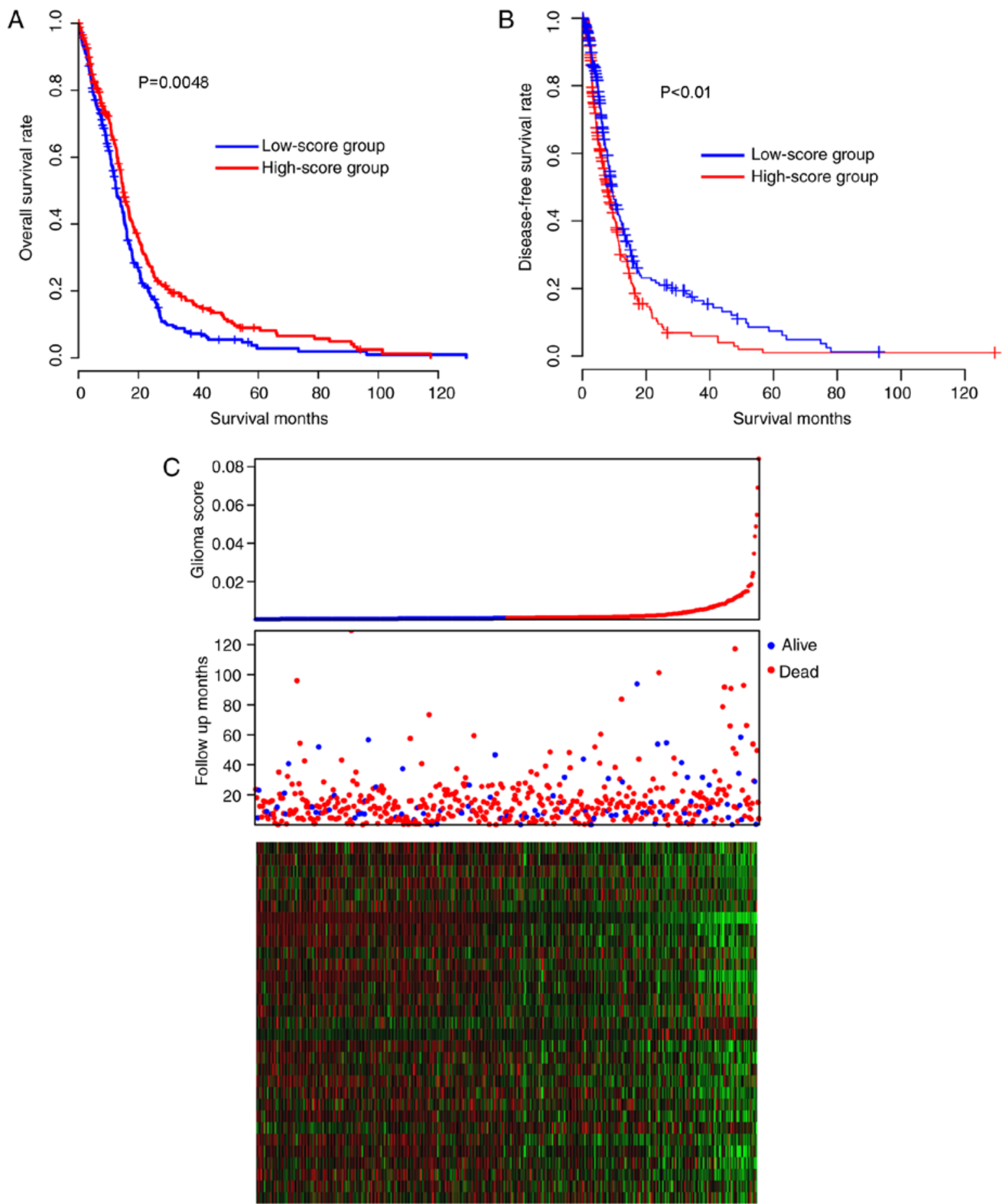

Figure 1. Performance of glioma scores in the training cohort. (A) The overall survival rate and (B) disease-free survival rate difference between the high- and low-score groups were depicted. (C) The detailed glioma score, survival status and gene expression profile were depicted. $\mathrm{P}<0.01$.

individuals has also been reported (31). These data explained why single biomarkers have frequently failed to predict the clinical outcome of glioma. Previous studies have emphasized the multiple gene expression-based models for prognosis, and models for other cancer types have been developed $(15,32)$; For example, Mammaprint has been reported as a powerful tool for therapy decision of breast cancer and OncotypeDX has been used in many types of cancer $(18,20)$, including ovarian cancer, breast cancer and colorectal cancer; as such, the model appears to be a successful predictor in independent cohorts using different platforms.
One limitation of the present study is that the detailed clinical information, including treatment and time to recurrence, which were critical for determining the prognosis of glioma, was unknown in all of the cohorts. Additionally, the surgical technique (R0/R1) is also not described in the clinical records. Another limitation is that the genes used for the calculation of glioma scores were a relatively large panel; however, it was considered that the panel is relatively small, compared with the transcriptome, and a large panel may reflect the biological status of glioma with greater accuracy. 

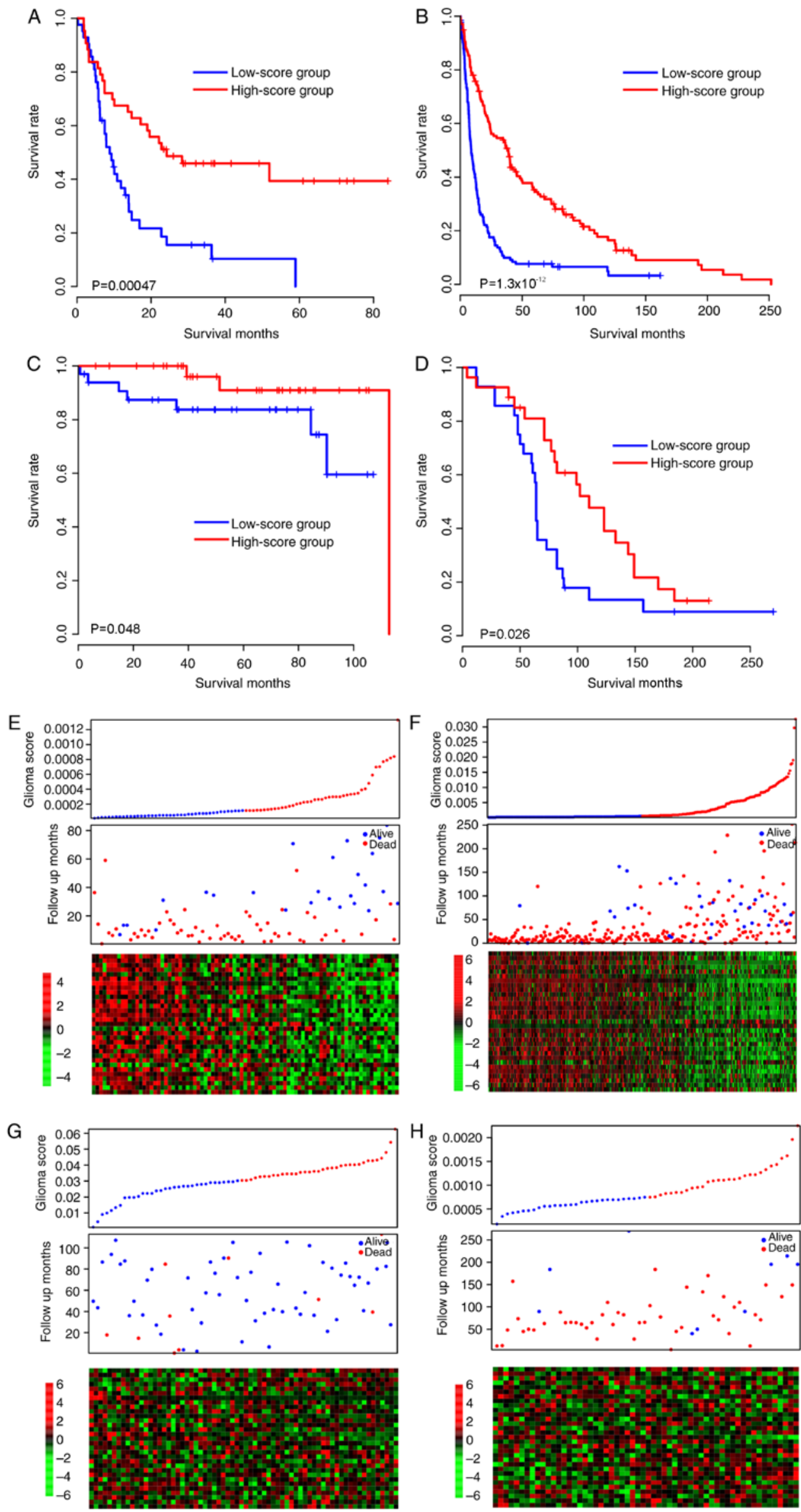

Figure 2. Robustness of the glioma score. The performance of the signature was also assayed for the (A) UCLA (GSE4412), (B) Netherland (GSE16011), (C) NPTB (GSE16581) and (D) Seoul cohorts (GSE42669), and the overall survival and expression pattern of (E) GSE4412, (F) GSE16011, (G) GSE16581, (H) GSE42669 were also presented. $\mathrm{P}<0.05$. The $\mathrm{x}$-axis is survival time (months), the $\mathrm{y}$-axis is the overall survival rate. 
A

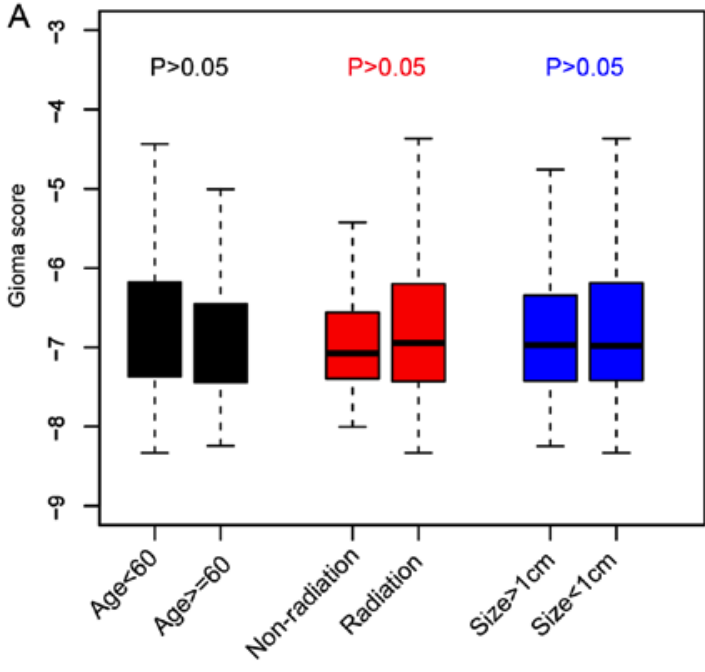

B

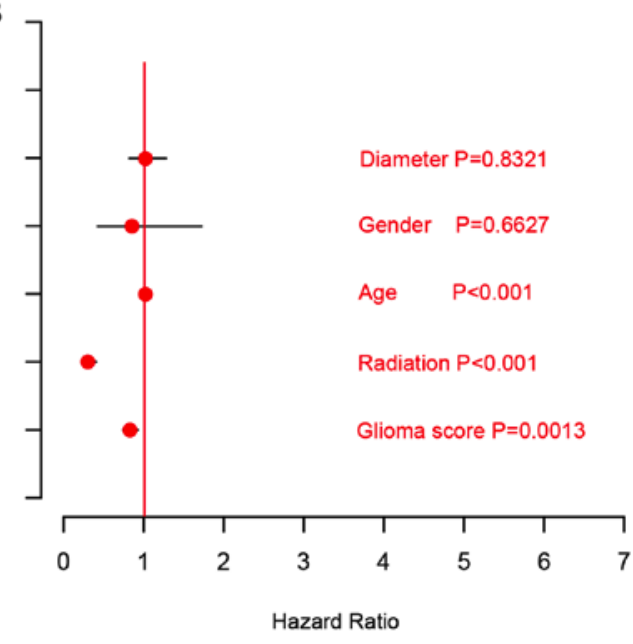

C

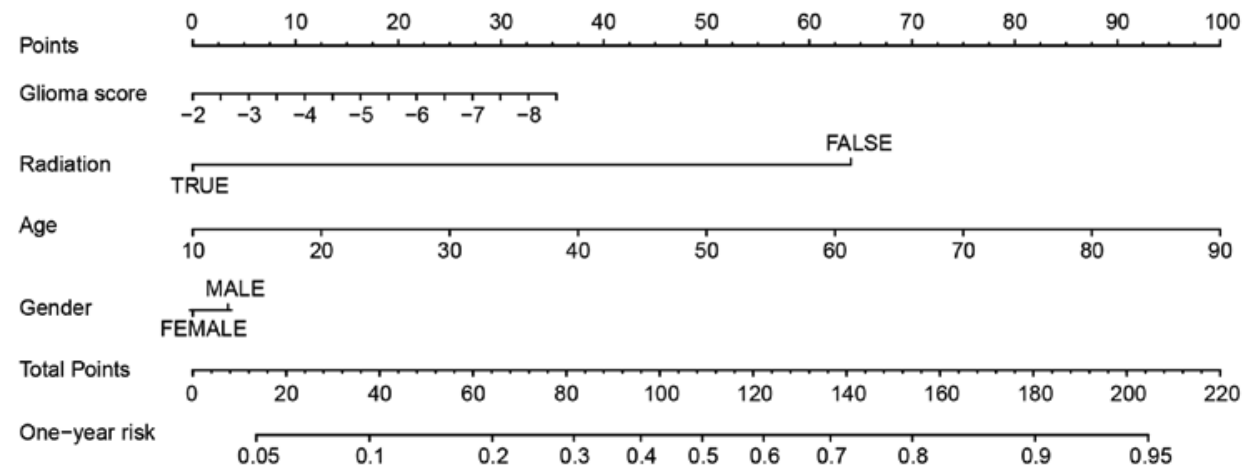

Figure 3. Clinical information and glioma scores. (A) The scores are not significantly associated with the clinical indicators, age, radiation and tumor size. (B) Cox multivariate regression and nomogram demonstrated that it is an important indicator, and (C) a nomogram indicating the clinical utilization of glioma scores along with other clinical indicators. $\mathrm{P}<0.05$.
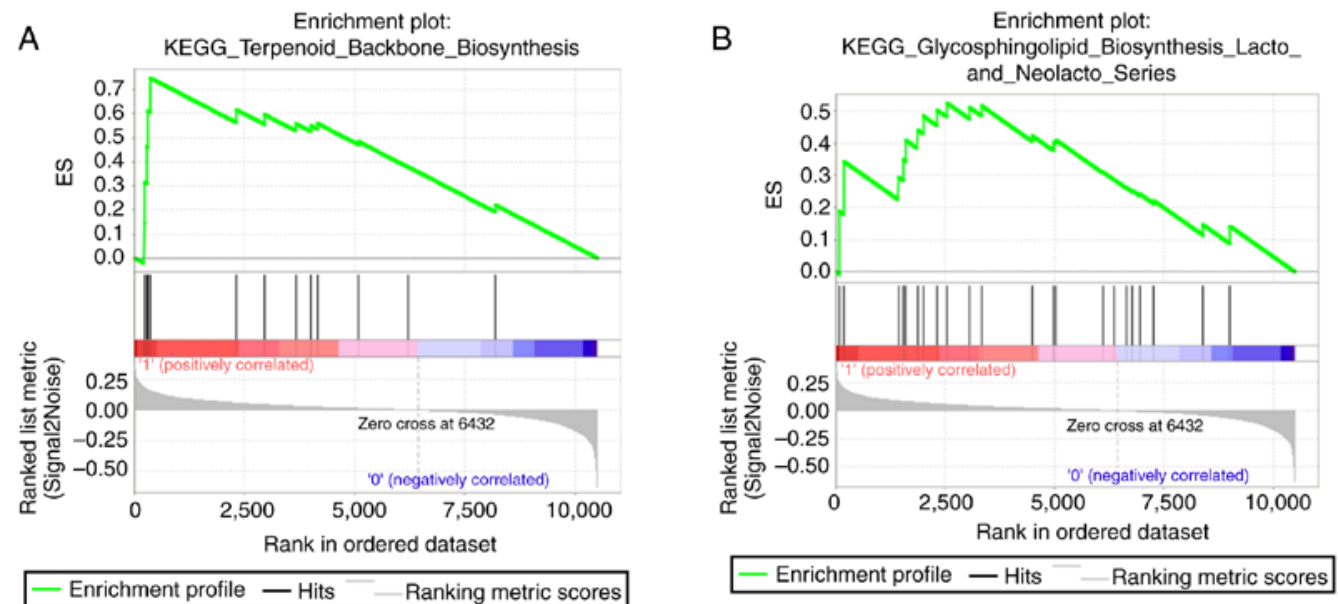

Figure 4. Glioma score-associated pathways. (A and B) The pathways significantly associated with glioma score were identified using Gene Set Enrichment Analysis, including (A) Terpenoid Backbone Biosynthesis and (B) Glycosphingolipid Biosynthesis. ES, enrichment score; KEGG, Kyoto Encyclopedia of Genes and Genomes. $\mathrm{P}<0.05$.

\section{Acknowledgements}

Not applicable.

\section{Funding}

No funding was received.

\section{Availability of data and materials}

The data are available from the Gene Expression Omnibus (https://www.ncbi.nlm.nih.gov/geo) with the provided accession numbers of Gene Expression Omnibus (accession nos. GSE4412, GSE16011, GSE16581 and GSE42669). 


\section{Authors' contributions}

ZY and JY assisted in the experimental design. LF, DX and YH assisted in data analysis. ZY, JY, LF, DX and YH were responsible for the proofreading and the writing of the present manuscript. All authors have read and approved the current version of the manuscript.

\section{Ethics approval and consent to participate}

Not applicable.

\section{Patient consent for publication}

Not applicable.

\section{Competing interests}

The authors declare that they have no competing interests.

\section{References}

1. Ostrom QT, Bauchet L, Davis FG, Deltour I, Fisher JL, Langer CE, Pekmezci M, Schwartzbaum JA, Turner MC, Walsh KM, et al: The epidemiology of glioma in adults: A 'state of the science' review. Neuro oncol 16: 896-913, 2014.

2. Chen W, Zheng R, Baade PD, Zhang S, Zeng H, Bray F, Jemal A, Yu XQ and He J: Cancer statistics in China, 2015. CA Cancer J Clin 66: 115-132, 2016.

3. Coppola D, Balducci L, Chen DT, Loboda A, Nebozhyn M, Staller A, Fulp WJ, Dalton W, Yeatman T and Brem S: Senescence-associated-gene signature identifies genes linked to age, prognosis, and progression of human gliomas. J Geriatr Oncol 5: 389-399, 2014.

4. Chen JW, Zhou CF and Lin ZX: The influence of different classification standards of age groups on prognosis in high-grade hemispheric glioma patients. J Neurol Sci 356: 148-152, 2015.

5. Cancer Genome Atlas Research Network: Comprehensive genomic characterization defines human glioblastoma genes and core pathways. Nature 455: 1061-1068, 2008.

6. Cancer Genome Atlas Research Network; Brat DJ, Verhaak RG, Aldape KD, Yung WK, Salama SR, Cooper LA, Rheinbay E, Miller CR, Vitucci M, Morozova O, et al: Comprehensive, integrative genomic analysis of diffuse lower-grade gliomas. N Engl J Med 372: 2481-2498, 2015.

7. Yan H, Parsons DW, Jin G, McLendon R, Rasheed BA, Yuan W, Kos I, Batinic-Haberle I, Jones S, Riggins GJ, et al: IDH1 and IDH2 mutations in gliomas. N Engl J Med 360: 765-773, 2009.

8. Esmaeili M, Hamans BC, Navis AC, van Horssen R, Bathen TF, Gribbestad IS, Leenders WP and Heerschap A: IDH1 R132H mutation generates a distinct phospholipid metabolite profile in glioma. Cancer Res 74: 4898-4907, 2014.

9. Shibahara I, Sonoda Y, Kanamori M, Saito R, Yamashita Y, Kumabe T, Watanabe M, Suzuki H, Kato S, Ishioka C and Tominaga T: IDH $1 / 2$ gene status defines the prognosis and molecular profiles in patients with grade III gliomas. Int J Clin Oncol 17: 551-561, 2012.

10. Thomas L, Di Stefano AL and Ducray F: Predictive biomarkers in adult gliomas: the present and the future. Curr Opin Oncol 25: 689-694, 2013

11. Cohen AL and Colman H: Glioma biology and molecular markers. Cancer Treat Res 163: 15-30, 2015.

12. Wang HJ, Gao Y, Chen L, Li YL and Jiang CL: RAB34 was a progression- and prognosis-associated biomarker in gliomas. Tumour Biol 36: 1573-1578, 2015.

13. Cai H, Xue Y, Liu W, Li Z, Hu Y, Li Z, Shang X and Liu Y: Overexpression of roundabout4 predicts poor prognosis of primary glioma patients via correlating with microvessel density. J Neurooncol 123: 161-169, 2015.
14. Gao H, Zhao H and Xiang W: Expression level of human miR-34a correlates with glioma grade and prognosis. J Neurooncol 113: 221-228, 2013

15. Wu X, Weng L, Li X, Guo C, Pal SK, Jin JM, Li Y, Nelson RA, Mu B, Onami SH, et al: Identification of a 4-microRNA signature for clear cell renal cell carcinoma metastasis and prognosis. PloS One 7: e35661, 2012.

16. Bou Samra E, Klein B, Commes T and Moreaux J: Identification of a 20-gene expression-based risk score as a predictor of clinical outcome in chronic lymphocytic leukemia patients. Biomed Res Int 2014: 423174, 2014.

17. Liu Q, Diao R, Feng G, Mu X and Li A: Risk score based on three mRNA expression predicts the survival of bladder cancer. Oncotarget 8: 61583-61591, 2017.

18. Cardoso F, van't Veer LJ, Bogaerts J, Slaets L, Viale G, Delaloge S, Pierga JY, Brain E, Causeret S, DeLorenzi M, et al: 70-Gene signature as an aid to treatment decisions in early-stage breast cancer. N Engl J Med 375: 717-729, 2016.

19. Marisa L, de Reynies A, Duval A, Selves J, Gaub MP, Vescovo L, Etienne-Grimaldi MC, Schiappa R, Guenot D, Ayadi M, et al: Gene expression classification of colon cancer into molecular subtypes: Characterization, validation, and prognostic value. PLoS Med 10: e1001453, 2013.

20. You YN, Rustin RB and Sullivan JD: Oncotype DX((R)) colon cancer assay for prediction of recurrence risk in patients with stage II and III colon cancer: A review of the evidence. Surg Oncol 24: 61-66, 2015.

21. Lee Y, Liu J, Patel S, Cloughesy T, Lai A, Farooqi H, Seligson D, Dong J, Liau L, Becker D, et al: Genomic landscape of meningiomas. Brain Pathol 20: 751-762, 2010.

22. Gravendeel LA, Kouwenhoven MC, Gevaert O, de Rooi JJ, Stubbs AP, Duijm JE, Daemen A, Bleeker FE, Bralten LB, Kloosterhof NK, et al: Intrinsic gene expression profiles of gliomas are a better predictor of survival than histology. Cancer Res 69: 9065-9072, 2009.

23. Freije WA, Castro-Vargas FE, Fang Z, Horvath S, Cloughesy T, Liau LM, Mischel PS and Nelson SF: Gene expression profiling of gliomas strongly predicts survival. Cancer Res 64: 6503-6510, 2004.

24. Joo KM, Kim J, Jin J, Kim M, Seol HJ, Muradov J, Yang H, Choi YL, Park WY, Kong DS, et al: Patient-specific orthotopic glioblastoma xenograft models recapitulate the histopathology and biology of human glioblastomas in situ. Cell Rep 3: 260-273, 2013.

25. Subramanian A, Tamayo P, Mootha VK, Mukherjee S, Ebert BL, Gillette MA, Paulovich A, Pomeroy SL, Golub TR, Lander ES and Mesirov JP: Gene set enrichment analysis: A knowledge-based approach for interpreting genome-wide expression profiles. Proc Natl Acad Sci USA 102: 15545-15550, 2005.

26. Ishwaran $\mathrm{H}$ and Kogalur UB: Consistency of random survival forests. Stat Probab Lett 80: 1056-1064, 2010.

27. Ishwaran H, Gerds TA, Kogalur UB, Moore RD, Gange SJ and Lau BM: Random survival forests for competing risks. Biostatistics 15: 757-773, 2014.

28. Robin X, Turck N, Hainard A, Tiberti N, Lisacek F, Sanchez JC and Müller M: pROC: An open-source package for $\mathrm{R}$ and $\mathrm{S}^{+}$to analyze and compare ROC curves. BMC Bioinformatics 12: 77, 2011 .

29. Li Z, Yan X, Sun Y and Yang X: Expression of ADP-ribosyltransferase 1 is associated with poor prognosis of glioma patients. Tohoku J Exp Med 239: 269-278, 2016.

30. Xu X, Wei Y, Wang S, Luo M and Zeng H: Serine-arginine protein kinase 1 (SRPK1) is elevated in gastric cancer and plays oncogenic functions. Oncotarget 8: 61944-61957, 2017.

31. Morokoff A, Ng W, Gogos A and Kaye AH: Molecular subtypes, stem cells and heterogeneity: Implications for personalised therapy in glioma. J Clin Neurosci 22: 1219-1226, 2015.

32. Jamshidi N, Jonasch E, Zapala M, Korn RL, Aganovic L, Zhao H, Tumkur Sitaram R, Tibshirani RJ, Banerjee S, Brooks JD, et al: The radiogenomic risk score: Construction of a prognostic quantitative, noninvasive image-based molecular assay for renal cell carcinoma. Radiology 277: 114-123, 2015.

This work is licensed under a Creative Commons Attribution-NonCommercial-NoDerivatives 4.0 International (CC BY-NC-ND 4.0) License. 